\title{
Importance des phases colloïdales et particulaires présentes dans les environnements marins
}

\author{
V. MOULIN*
}

révisé le 10 novembre 1994 , accepté le 19 janvier 1995)

RÉSUMÉ Cet article constitue une revue bibliographique sur les phases colloïdales et particulaires présentes dans les eaux marines (nature, importance pondérale, caractéristiques, et techniques analytiques associées) et sur leur effet sur la distribution de radionucléides (ou analogues) dans l'environnement marin. La première partie fait le point sur les techniques utilisées (essentiellement la microscopie électronique à transmission) pour la caractérisation de ces phases dans le milieu marin. De cette étude, il ressort d'autres axes d'études à privilégier. La seconde partie montre l'importance de ces phases collö̈dales et particulaires (même si leur importance pondérale est faible) dans la répartition des éléments en trace dans les environnements marins. Notamment, les travaux soulignent, outre l'importance des phénomènes de sorption aux interfaces des colloïdes/particules, le rôle non négligeable des phénomènes tels que les interactions particules-particules (ou colloïdes-colloïdes) conduisant aux processus de coagulation/sédimentation. La compréhension de l'ensemble des phénomènes est basée sur des approches expérimentales complémentaires : étude de systèmes modèles, étude de systèmes naturels (eaux de mer, eaux côtières).

ABSTRACT This paper constitutes a bibliographic review on the colloidal and particulate phases occurring in marine waters (nature, weighable importance, characteristics and associated analytical techniques) and their impact on radionuclide (or analogue) distribution in the marine environment. The first part points out the different techniques used to characterise the colloidal and particulate phases (in particular, transmission electron microscopy) in marine systems. From this study, other research areas are underlined. The second part shows the importance of the colloidal and particulate phases (even if their weighable importance is low) on the distribution of trace elements in marine systems. In particular, the studies underline, besides the importance of sorption phenomena at colloid/particle interfaces, the major role of phenomena such as the particle/particle (or colloid/colloid) interactions leading to coagulation/sedimentation processes. The understanding of the whole phenomena relies on complementary experimental approaches such as study of model and natural systems (marine or coastal waters).

\footnotetext{
* Commissariat à l'énergie atomique, Centre d'études de Fontenay-aux-Roses, Direction du cycle du combustible, DESD/SESD/Section de géochimie, BP 6, 92265 Fontenay-aux-Roses Cedex, France.
} 


\section{Introduction}

Le devenir d'éléments radiotoxiques, en particulier les éléments de longue période (cas des éléments transuraniens), dans les systèmes aquatiques naturels quels qu'il soient (milieu marin ou terrestre) est particulièrement important à connaître, notamment vis-à-vis de leur biodisponibilité. Pour ce faire, la connaissance des formes sous lesquelles ces éléments radiotoxiques sont présents en solution (c'est-à-dire déterminer leur spéciation) est essentielle et, plus particulièrement, leur distribution vis-à-vis des entités présentes (autres que dissoutes) dans le milieu aquatique : matières organiques, colloïdes, particules.

Ces collö̈des définis comme des particules de taille comprise entre le nanomètre et le micromètre (les particules présentant alors des tailles supérieures au micromètre ${ }^{1}$ ) sont présents en suspension dans l'eau et possèdent des propriétés spécifiques telles que des propriétés interfaciales, des propriétés de piégeage vis-à-vis de polluants inorganiques et organiques. Ces colloïdes, de nature organique (substances humiques, bactéries, microorganismes) et inorganique (silicates, silicoaluminates, oxyhydroxydes métalliques,...), peuvent être identifiés dans les milieux aquatiques (eaux douces, marines) par différentes techniques analytiques permettant d'atteindre leur composition, leur taille et leur concentration. De par leurs propriétés, ces colloïdes peuvent modifier la distribution de cations dans des systèmes naturels et, de ce fait, changer leur devenir : en particulier dans des milieux fracturés ou poreux, ils peuvent soit accélérer la migration d'éléments radiotoxiques (par leur aptitude à piéger des cations), soit retarder leur mobilité par des effets de filtration (dus à leur taille), de sorption (rétention sur des surfaces minérales) ou encore de floculation-sédimentation (dû à des effets de charge) [18].

Dans des systèmes ouverts (fleuves, océans, mers), l'effet des colloïdes et des particules sur le devenir d'éléments polluants (dont les radiotoxiques) est quelque peu différent ; certains de ces processus seront mineurs voire quasiinexistants (filtration par le milieu) et d'autres seront prépondérants (sorption à la surface des colloïdes/particules et maintien en suspension, floculation et sédimentation des colloïdes et des particules, interaction avec les sédiments).

C'est sur ces derniers systèmes, en particulier le milieu marin, que nous focaliserons le contenu de cet article. La question qui se pose est de savoir si les colloïdes et les particules sont importants à prendre en compte dans un tel système. D'une part, en raison de leur réactivité vis-à-vis d'éléments toxiques, d'autre part en raison de leur temps de résidence dans les eaux (dû à leur taille), les éléments piégés par ces entités auront un temps de résidence imposé par celui des particules [34]. Ceci nécessite de connaître le rôle de ces collö̈des et particules sur la distribution d'éléments radiotoxiques dans le milieu marin.

1. Certains auteurs définissent la fraction colloïdale comme étant la fraction $1 \mathrm{~nm}-400 \mathrm{~nm}$ (seuil de coupure de certains filtres). 
Le but de cet article est d'effectuer une revue sur :

- les phases colloïdales et particulaires présentes dans les eaux marines: nature, importance pondérale, caractéristiques, techniques analytiques associées et

- leur effet sur la distribution de radionucléides (ou analogues) dans ces systèmes.

\section{Phases colloïdales et particulaires présentes dans les eaux marines}

\subsection{Techniques analytiques}

Les principales techniques utilisées pour caractériser les phases colloïdales et particulaires dans les eaux (quel que soit leur environnement hormis les eaux marines) sont répertoriées dans [18]. Le but de ces techniques est d'obtenir sur les colloïdes et les particules présents le maximum d'informations pertinentes dans le cadre des études liées avec le comportement d'éléments radiotoxiques (ou polluants) dans les systèmes naturels.

Ces caractéristiques sont les suivantes :

- taille et distribution en taille : important pour prévoir leur éventuelle aptitude à migrer ou à sédimenter ;

- composition et nature: important pour connaître leur genèse en liaison avec leur environnement géologique et géochimique, pour prévoir le comportement de certains éléments au moyen des codes de calcul ;

- morphologie : important pour permettre de classifier entre phases néoformées et phases héritées (liées aux mécanismes de formation des colloïdes, respectivement à partir de la solution ou à partir d'une phase solide) ;

- abondance (en nombre ou en masse) : important pour être à même d'utiliser ces valeurs dans des codes géochimiques et pour estimer leur importance par rapport à d'autres systèmes de référence.

Il est également important de pouvoir déterminer la concentration en éléments en traces associés à ces phases et témoignant ainsi de leur aptitude à modifier la distribution des éléments aux interfaces eau-solide. Dans ce cas, des techniques de type destructif sont le plus souvent employées pour atteindre la concentration en éléments traces associés à une phase colloïdale, particulaire ou dissoute (exemple : analyse chimique du filtrat, du rétentat après digestion acide).

Les principales techniques sont rassemblées dans le tableau I. Elles sont classées i) selon le type d'échantillon produit : analyse des phases colloïdales ou particulaires directement dans leur milieu d'origine, ou concentrées sur un filtre et ii) selon le type d'informations obtenu (analyse discrète ou globale). De façon synthétique, les techniques les plus couramment utilisées pour une caractérisation des colloïdes sont au nombre de quatre. 
TABLEAU I

Principales techniques mises en ceuvre pour l'étude des colloïdes et particules Main techniques used for the study of colloids and particles

\begin{tabular}{|c|c|c|}
\hline Techniques & $\begin{array}{l}\text { Analyse discrète : } \\
\text { dans la phase fluide ou } \\
\text { déposées sur un filtre } \\
\text { (ou autre support) }\end{array}$ & $\begin{array}{l}\text { Analyse globale: } \\
\text { sur la phase fluide } \\
\text { (concentré, filtrat, ...) } \\
\text { ou après digestion } \\
\text { de la membrane }\end{array}$ \\
\hline Diffusion de lumière & $\mathbf{x}$ & 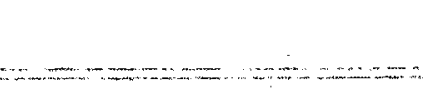 \\
\hline $\begin{array}{l}\text { Microscopies } \\
\text { électroniques (MEB ou MET) }\end{array}$ & $\mathbf{x}$ & \\
\hline Electrophorèse & $\mathbf{x}$ & \\
\hline Ultracentrifugation & 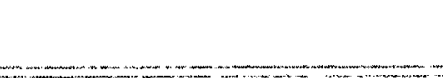 & $\mathrm{x}$ \\
\hline Analyse du carbone organique & & $\mathbf{x}$ \\
\hline Gravimétrie & $\mathrm{x}$ & \\
\hline $\begin{array}{l}\text { ICP-MS/AES, fluorescence, } \\
\text { activation neutronique }\end{array}$ & & $\mathrm{x}$ \\
\hline
\end{tabular}

- La microscopie électronique à balayage (MEB) permet d'étudier la morphologie et la granulométrie (avec, par exemple, une limite de détection à $15 \mathrm{kV}, 39 \mathrm{~mm}$ de distance d'observation : $70 \mathrm{~nm}$ ) des collö̈des le plus généralement concentrés par filtration ou ultrafiltration transversale (définir le volume à filtrer, la dimension du filtre, la nature du filtre - inorganique ou organique - ainsi que sa porosité) sur un filtre (séché puis métallisé pour l'examen au MEB); les techniques de préparation des filtres sont répertoriées dans [4] dans le cas d'eaux douces.

- La microscopie à transmission (MET) permet l'examen morphologique, structural (grâce aux clichés de diffraction) et l'analyse granulométrique des colloïdes déposés sur une grille; les différentes techniques de préparation d'échantillons sont répertoriées dans $[17,21,24,31]$ pour des eaux douces.

Si ces deux outils sont couplés à un système d'analyse dispersif, ils permettent également d'effectuer une microanalyse des particules et d'avoir ainsi accès à leur composition (mais non quantitative) ; d'autre part, ces deux techniques permettent d'effectuer un dénombrement des particules (effectué manuellement ou au moyen d'un analyseur d'images).

- La spectroscopie de corrélation de photons permet une analyse granulométrique jusqu'à quelques nanomètres (fonction de la concentration) sur une eau brute ou filtrée (aspect non-destructif par rapport à la microscopie). 
- La filtration couplée à la gravimétrie permet d'accéder à la concentration massique en colloïdes ou en particules correspondant à une classe granulométrique donnée (selon la porosité du filtre choisi).

\subsection{Méthodes de prélèvement}

Les travaux $[16,31-33]$ portant sur les eaux de mer font état de l'utilisation de flacons en téflon ou en verre, de l'ajout de chlorure mercurique pour préserver les échantillons $(1 \mu \mathrm{M}$ de $\mathrm{Hg})$. Les eaux sont stockées à l'abri de la lumière et à une température de quelques degrés. Il a été vérifié par Wells et Goldberg [31] que l'ajout de chlorure mercurique $\left(\mathrm{HgCl}_{2}\right)$ aux échantillons ne perturbait pas l'analyse ultérieure, en particulier les résultats sur la distribution en taille et sur la concentration en colloïdes. Cependant, des résultats récents [23] ont montré que l'ajout d'un antibactérien $\left(\mathrm{HgCl}_{2} \quad 0,04-0,4 \mathrm{mM}\right.$ ou $\mathrm{NaN}_{3}$ 0,1-15 mM) dans une eau de rivière (Rhin) n'empêchait pas le développement bactérien au-delà de 5-7 jours. L'utilisation de concentrations supérieures n'est pas possible en raison d'une précipitation du chlorure mercurique ou de l'interférence de l'azide de sodium lors de l'analyse de carbone organique en solution.

\subsection{Préparation des échantillons}

L'essentiel des travaux sur les phases colloïdales et particulaires des eaux de mer a été réalisé par Wells et Goldberg [31-33] qui ont principalement utilisé la microscopie électronique à transmission (MET) comme technique analytique pour déterminer la taille, la nature et la concentration (en nombre) des colloïdes. Les travaux de Lavoie [16] à caractère plus méthodologique (développement d'une méthode combinant la microscopie électronique à balayage (MEB), la microanalyse et l'analyse d'image automatisée) font également état de données sur la taille et la nature des colloïdes marins. Les études de BrunCottan et al. [4] sont très spécifiques et portent exclusivement sur la calcite.

\section{Cas de l'analyse par MEB}

\section{Concentration des particules sur un filtre}

Les échantillons d'eau de mer brute sont filtrés $(250 \mathrm{ml})$ sur des membranes en polycarbonate (telles que Nuclépore $^{2}$ ) de porosité $0,4 \mu \mathrm{m}$ (diamètre des filtres $25 \mathrm{~mm}$ ) sous une pression de 1 bar [16]. Les filtres sont ensuite conservés sur des blocs de cellulose saturés en glutaraldéhyde $(2 \%$ dans de l'eau de mer filtrée) dans des boîtes appropriées à une température de $4{ }^{\circ} \mathrm{C}$.

Des études plus conventionnelles [4] utilisent des filtres Nuclépore et soulignent i) l'importance du rinçage des filtres par de l'eau millipore pour éviter la présence de gypse (par recristallisation des sels résiduels), ce qui entraîne un artefact considérable pour l'analyse du calcium, et ii) la rapidité du rinçage du filtre pour éviter la dissolution de petites particules de carbonate de calcium.

2. Filtre en polycarbonate à structure fine (pores obtenus par bombardement de particules de la matrice). 


\section{Préparation du filtre pour l'examen au $M E B$}

Le filtre subit une préparation à base de $\mathrm{RuO}_{4}{ }^{3}(0,5 \%)$, est dessalé, rincé et déshydraté. Le but de cette opération très spécifique est d'augmenter la densité électronique des particules organiques en les imprégnant d'un élément lourd de façon à obtenir une image binaire plus aisément gérable pour l'analyse automatique développée par [16]. Cette technique est d'usage courant en biologie pour préparer des échantillons au MET. La présence de Ru ne perturbe pas la microanalyse car la raie $L \alpha$ du ruthénium interfère seulement avec le chlore (raie $\mathrm{K} \alpha$ ), ce qui n'est pas préjudiciable pour l'analyse.

\section{Examen au $M E B$}

Un détecteur d'électrons rétrodiffusés est utilisé dans le cas de l'étude de [13]. La limite de détection obtenue dans les conditions de l'analyse (tension, distance de travail non spécifiées par l'auteur) est de $1 \mu \mathrm{m}$. L'acquisition lors de la microanalyse est de $2 \mathrm{~s}$.

\section{Cas de l'analyse par MET}

\section{Préparation de la grille pour l'examen au MET}

Les échantillons pour l'analyse au MET sont produits directement sur le lieu de prélèvement. Les particules sont déposées sur une grille (spécifique pour l'analyse au MET et rendue hydrophile par un traitement spécifique [32]) par ultracentrifugation directement dans les tubes spécialement adaptés pour cette opération. Les conditions de centrifugation sont choisies pour séparer des particules selon leur densité et leur taille. Wells et Goldberg [32] ont choisi : vitesse $41000 \mathrm{rpm}$, temps $4 \mathrm{~h}$ (permet de récupérer des particules de taille de l'ordre de $15 \mathrm{~nm}$ et plus pour une densité minimale de $1,22 \mathrm{~g} / \mathrm{cm}^{3}$ ). Le volume d'eau de mer brute traité est de $10 \mathrm{ml}$. Le surnageant est alors retiré et la grille est rincée avec de l'eau distillée filtrée, puis séchée au dessiccateur. Le rendement d'une telle opération est de $\sim 95 \%$ (calculé sur des suspensions colloïdales standards).

\subsection{Résultats}

Il résulte des travaux 4 [4, 9-10, 16, 26, 31-32] les conclusions suivantes sur la nature, l'abondance et la taille des colloïdes "marins" :

- Nature des colloïdes et des particules identifiés :

- organique:

i) organismes vivants tels que bactéries, virus (facilement identifiables car relativement transparents sur les photographies en microscopie électronique) ; faible signal en microanalyse (excepté des traces de P),

ii) matière organique détritique (pas de structure, pas de signal en microanalyse) qui constitue la majeure partie des colloïdes "organiques";

3. Produit très toxique.

4. Travaux portant sur la mer des Sargasses, sur l'océan Pacifique (Nord-Ouest, sub-tropical). 
- inorganique:

i) des colloïdes de fer avec des tailles comprises entre 100 et $500 \mathrm{~nm}$ (voire plus), présents sous forme d'agrégats de particules ayant des formes elliptiques,

ii) des collö̈des argileux (aluminosilicates) avec des tailles variant entre 200 et $1000 \mathrm{~nm}$, $8 \mu \mathrm{m}$,

iii) des particules riches en $\mathrm{Si}, \mathrm{Ca}, \mathrm{S}$ avec des tailles comprises entre 0,5 et

iv) des particules de calcite de quelques $\mu \mathrm{m}$.

D'une façon générale, il s'avère que les colloïdes organiques constituent la majeure partie des phases colloïdales présentes dans les eaux de mer (travaux aux MEB et MET; analyses du carbone organique colloïdal). En particulier, les travaux de Guo et al. [10] relatifs au Golfe du Mexique montrent que la fraction colloïdale organique supérieure à 1000 daltons (ou encore $1 \mathrm{~nm}^{5}$ ) constitue en moyenne $45 \%$ du carbone organique dissous $(<0,4 \mu \mathrm{m})^{6}$ et la fraction colloïdale supérieure à 10000 daltons (ou encore $1,5 \mathrm{~nm}$ ) seulement 11 $\%$. Une compilation des données concernant le carbone colloïdal organique dans les eaux marines [10] montre que cette fraction peut représenter entre quelques pourcent (pour des eaux de mer d'origine non spécifiée) et presque $50 \%$ (Golfe du Mexique) du carbone organique dissous. Dans le cas de l'Atlantique, elle en représente 8 à $16 \%$.

L'origine de ces colloïdes organiques semble être essentiellement autochtone même si certains auteurs rapportent une origine terrestre [8]. Les matières organiques d'origine terrestre représentent une faible proportion de la matière organique totale présente dans l'océan.

- Morphologie des colloüdes: observation de grappes constituées de granules de taille $2-5 \mathrm{~nm}$ pour la fraction granulométrique $<60 \mathrm{~nm}$ et coalescence de ces grappes pour la fraction $60-200 \mathrm{~nm}$.

- Taille des colloïdes : l'ensemble des travaux montre, selon les techniques d'observation utilisées, une très grande distribution en taille des entités présentes dans les eaux de mer. Les travaux au MET montrent une présence de colloïdes de taille inférieure à 120 nm qui constituent la fraction la plus abondante. Les travaux résultant de l'étude au MEB montrent des colloïdes de taille supérieure à $0,5 \mu \mathrm{m}$ (voire jusqu'à quelques $\mu \mathrm{m}$ ) présents à une concentration de 500 à $310^{3} \mathrm{part} / \mathrm{ml}$ ou de 30-100 $\mu \mathrm{g} / \mathrm{l}$. Dans le cas d'études spécifiquement liées au carbone organique colloïdal, les tailles sont très faibles et les auteurs parlent préférentiellement de poids moléculaire (1 000 ou 10000 daltons).

5. L'équivalence dalton - nanomètre est basée sur la calibration de filtres avec des protéines globulaires (données Amicon).

6. Dans ces travaux, les auteurs définissent la fraction colloïdale comme étant comprise entre le nanomètre et 0,4 micromètre. Le carbone organique dissous (COD) représente la teneur en carbone après filtration à $0,45 \mu \mathrm{m}$. 
- Concentration en colloïdes :

i) Variation selon la profondeur du prélèvement, entre $<10^{4}$ particules $/ \mathrm{ml}$ (limite de détection en surface) et $10^{9}$ particules $/ \mathrm{ml}$ (entre 30 et $100 \mathrm{~m}$ ) pour les particules de taille inférieure à $120 \mathrm{~nm}$; ces concentrations varient selon la période du prélèvement; les concentrations les plus élevées sont observées dans la région du "thermocline" (constaté pour d'autres zones de prélèvement), et les concentrations les plus basses (limite de détection) pour les zones les plus profondes; cette fraction serait en partie constituée par des colloïdes de nature organique $[4,31-33]$.

Quelques auteurs ont proposé des modèles pour tenter d'expliquer cet effet de concentration en fonction de la profondeur : modèle de transport de particules "horizontal" d'advection/diffusion ou modèle "vertical" de sédimentation [4]. Des considérations physiques, biochimiques et sédimentologiques amènent à penser que le modèle "horizontal" est le plus adapté (dans les cas des particules de calcite). Ce modèle implique alors un flux permanent de carbonates du réservoir sédimentaire profond vers l'océan profond et ceci entraîne donc la présence de particules en suspension tant que le réservoir n'est pas épuisé.

ii) Augmentation de la concentration (en nombre) lorsque leur taille (fraction $<120 \mathrm{~nm}$ ) diminue (variation logarithmique). Ceci a été observé pour des particules marines de taille supérieure au micromètre et également pour des collö̈des d'origine granitique [5]. Cependant, la loi logarithmique ne reproduit pas les résultats obtenus pour la fraction inférieure à $40 \mathrm{~nm}$, ce qui peut être attribué à une agrégation des plus petites particules. La morphologie des agrégats observés témoigne à la fois d'un régime diffusif et réactif qui indique la variation de la dynamique des colloïdes en milieu marin.

Les concentrations mentionnées dans la littérature varient a) en nombre, entre $10^{3}$ et $10^{9}$ particules $/ \mathrm{ml}$ et b) en masse, entre 10 et $100 \mu \mathrm{g} / \mathrm{l}$, voire plus. Des mesures plus spécifiques de la teneur en carbone organique ${ }^{7}$ dans les eaux de mer indiquent des teneurs de quelques mg/l (ou de l'ordre de quelques centaines de micromoles de carbone). Des études dans le golfe du Mexique [10] ont montré une distribution horizontale et verticale du carbone organique : une diminution de la teneur lors de l'éloignement des côtes (semblant indiquer un apport terrestre de carbone ou côtier) ainsi qu'un gradient vertical décroissant.

Pour référence, une eau granitique (Grimsel, Suisse) [5] analysée au laboratoire dans le cadre d'un contrat communautaire contient $10^{5}$ part./ml pour une classe granulométrique de $80-1000 \mathrm{~nm}$, ce qui, ramené à une concentration massique, correspond à quelques $200 \mu \mathrm{g} / \mathrm{l}$ avec une teneur en carbone organique total de $100 \mu \mathrm{g} / \mathrm{l}$.

7. Il faut noter la difficulté de mesure du carbone organique dans l'eau de mer. Ceci a fait l'objet de nombreux travaux tant sur le point méthodologique que sur le plan interprétation du cycle du carbone (voir références dans Guo et al.) [10]. 


\section{Rôle des phases colloïdales et particulaires présentes dans les eaux marines sur la distribution d'éléments en trace}

\subsection{Extension des recherches}

De nombreux travaux (laboratoire, terrain, modèles) montrent l'importance de ces phases colloïdales ou particulaires sur la distribution des éléments en trace dans les eaux marines $[1,2,7,11,13,15,19,25,27,30,34]$ pour les raisons suivantes :

- réactivité vis-à-vis d'éléments toxiques,

- indication sur le temps de résidence des éléments piégés.

Les questions qui se posent sont les suivantes :

- quel est le devenir dans le milieu marin des éléments en trace venant d'une rivière polluée (ou de retombées atmosphériques) ?

- quel est le temps de résidence des éléments piégés par la matière en suspension?

- le comportement des métaux en trace est-il conservatif ou non (sorption/désorption)?

Certaines de ces questions trouvent des éléments de réponse dans la littérature vis-à-vis d'études de laboratoire (étude de la désorption par exemple) ou d'études de terrain (répartition d'éléments en trace vis-à-vis des phases colloïdales et particulaires présentes) mais sont spécifiques de certains éléments $(\mathrm{Pb}$, $\mathrm{Cd}, \mathrm{Cu}, \mathrm{Th}, \mathrm{Zn}$, terres rares). Dans ces études, sont dissociés les éléments recyclés, c'est-à-dire ceux dont la concentration augmente avec la profondeur (tels $\mathrm{Ni}, \mathrm{Cu}, \mathrm{Zn}$ ), des éléments piégés, c'est-à-dire ceux dont la concentration diminue lorsque la profondeur augmente (tels $\mathrm{Pb}, \mathrm{Bi}, \mathrm{Co}, \mathrm{Th}$ ) $[8,12]$.

De nombreuses études liées aux éléments lourds (et analogues de radionucléides) portent sur le thorium et sur les terres rares, ce qui présente un certain intérêt dans le cadre des recherches qui nous concernent (en tant qu'analogues du plutonium tétravalent et des actinides trivalents).

\subsection{Cas du thorium}

Les travaux de Honeyman et Santschi [12] ont montré que $15 \%$ du thorium se trouve associé avec la matière colloïdale dans les milieux profonds et ceux de Baskaran et al. [1] ont montré que 10 à $78 \%$ du thorium est associé avec la fraction comprise entre $1 \mathrm{~nm}$ et $400 \mathrm{~nm}$ dans les eaux du Golfe du Mexique.

De plus, l'isotope 234 constitue en traceur in situ de la dynamique des particules (isotope descendant de ${ }^{238} \mathrm{U}$ qui, lui, est conservatif, sous forme de complexes carbonates dans les eaux marines). En effet, la distribution d'un élément (par exemple le $\mathrm{Th}$ ) entre l'ensemble des petites et grosses particules est contrôlée par les processus d'agrégation/désagrégation. Compte-tenu du fait 
que les petites particules $(0,4-10 \mu \mathrm{m})$ présentent de faibles vitesses de sédimentation (<1m/jour) par rapport aux grosses particules $(10-100 \mu \mathrm{m})$, le flux vertical de l'élément considéré sera essentiellement contrôlé par les interactions petite/grosse particule [1]. C'est le modèle de "pompage colloïdal" (passage d'une fraction colloïdale vers une fraction particulaire) qui comprend deux étapes :

i) une étape rapide correspondant à l'adsorption,

ii) une étape plus lente correspondant à l'agrégation des colloïdes.

Dans le cas de la première étape, la cinétique peut être rapide pour un système simple ( $M$-particule ou site réactionnel) mais pour un système multiligand la cinétique peut devenir plus lente [15].

La dynamique des particules dans les océans correspond à un ensemble compliqué de processus d'agrégation/désagrégation, ce qui amène à prendre en compte le processus de sédimentation. Par exemple, une suspension d'hématie (diamètre des particules $250 \mathrm{~nm}$ ) à $4 \mathrm{ppm}$ reste stable $55 \mathrm{~min}$, puis il y a coagulation et sédimentation; ce phénomène est d'autant plus important que la concentration initiale est élevée ou encore le système est d'autant plus stable vis-à-vis de la coagulation-sédimentation que la concentration en colloïdes est faible. Différents modèles sont proposés dans la littérature pour décrire la coagulation, en particulier des modèles semi-empiriques [15].

Il apparaît donc important de suivre le devenir des particules, en particulier l'étude des phénomènes de coagulation (le cation suivra ce processus). Ceci implique de connaître :

- les mécanismes de sorption,

- la réversibilité (modèles réversible ou irréversible proposés dans la littérature),

- les phénomènes de coagulation,

- les cinétiques (importantes dans le cas de phénomènes de coagulation).

D'autres auteurs soulignent également l'importance de l'activité biologique qui peut régir la distribution des particules en suspension en surface [30], ou encore être un processus contrôlant la sorption [13].

\subsection{Cas des terres rares}

La spéciation des terres rares dans les eaux de mer est essentiellement dominée par les complexes carbonates [27]. Néanmoins, ces auteurs ont constaté un fractionnement: les terres rares légères (La) sont préférentiellement absorbées sur les surfaces de particules marines (films organiques ou inorganiques - oxydes de manganèse)) alors que les terres rares lourdes (Lu) restent plutôt en solution. Ces résultats obtenus pour la mer des Sargasses sont similaires à ceux obtenus pour un autre océan (océan Indien) suggérant ainsi la non-spécificité des résultats (et, par là même, utilisation des constantes de com- 
plexation pour prédire la distribution d'un élément). Ce fractionnement entre l'eau de mer et les particules en suspension est un phénomène se produisant dans la zone de surface et un relarguage des terres rares est observé en profondeur (reminéralisation indiquant ainsi une association labile terre rare-particule).

De plus, cette étude [27] montre le rôle fondamental joué par les oxyhydroxides de Mn dans l'oxydation de Ce (III) dissous en Ce (IV) particulaire ainsi que dans le fractionnement des terres rares en général. Grâce aux données sur le cérium, des temps de résidence des particules dans la mer des Sargasses (environ $13 \mathrm{j}$ ) ont pu être calculés (en accord avec ceux obtenus par les isotopes du thorium : $18 \mathrm{j}$ ).

Des modèles sont utilisés dans la littérature pour décrire les interactions métal-particule, en particulier les modèles d'adsorption développés pour décrire les systèmes oxyde-métal comme le modèle de complexation de surface $[8,22]$. A partir des constantes de complexation de surface (déterminées au laboratoire sur des systèmes modèles), la distribution d'un élément donné peut-être calculée (prédite) et comparée par rapport à celle obtenue sur le système naturel (détermination de la répartition d'un élément par analyses chimiques).

Ces études portent sur les eaux marines mais certains auteurs soulignent l'importance des eaux côtières pour plusieurs raisons : [30] ;

- des concentrations plus fortes en colloïdes et particules ont été observées

- l'origine des particules relève de processus biologiques et sédimentaires (remise en suspension du sédiment) impliquant des variations saisonnières (selon l'origine, ces variations ne sont pas en phase);

- l'importance des phénomènes de floculation lorsque la salinité augmente (notamment près des estuaires) ; ces phénomènes sont importants à prendre en considération pour le devenir des colloïdes et particules et les éléments lourds associés ;

- des temps de résidence différents dans les zones côtières ont également été calculés.

Ces zones côtières représentent des systèmes relativement complexes en raison des différents processus qui y ont lieu.

\section{Conclusions}

Peu d'études ont été consacrées à la caractérisation sensu stricto des colloïdes et particules marins qui sont toutefois reconnus comme étant un possible piège d'éléments toxiques. Les travaux concernant le carbone organique dans les océans existent dans le cadre général d'études liées à la biomasse et au cycle de cet élément. Plusieurs axes d'études pourraient être intéressants à dévelop- 
per pour l'étude des phases colloïdales et particulaires dans le milieu marin :

- utilisation de la microscopie électronique à balayage (MEB) : cette technique plus aisée à mettre en ouvre (notamment pour la préparation des échantillons) n'a pas été systématique pour la caractérisation des colloïdes et particules dans les eaux marines;

- utilisation de la technique de spectroscopie de corrélation de photons (diffusion de lumière en mode dynamique) dans le but de caractériser la distribution en taille des entités présentes.

L'ensemble de ces techniques pourra contribuer à apporter des informations sur la nature et les caractéristiques des colloïdes et des particules présents dans les eaux marines.

Cette revue bibliographique montre l'importance de ces phases colloïdales et particulaires (même si leur importance pondérale est faible) dans la répartition des éléments en trace dans les environnements marins. Notamment, ces travaux soulignent, outre l'importance des phénomènes de sorption aux interfaces des colloïdes/particules, le rôle non négligeable des phénomènes tels que les interactions particules-particules (ou colloïdes-colloïdes) conduisant aux processus de coagulation/sédimentation. La compréhension de l'ensemble des phénomènes est basée sur des approches expérimentales complémentaires : étude de systèmes modèles, étude de systèmes naturels (eaux de mer, eaux côtières). Ces différentes approches constituent un axe important à conserver pour les études ultérieures à mener sur ces systèmes.

\section{Remerciements}

Ce travail a été réalisé grâce au soutien de l'Institut de protection et de sûreté nucléaire (IPSN) du Commissariat à l'énergie atomique.

\section{RÉFÉRENCES}

[1] BASKARAN M., SANTSCHI P.H., BENOIT G., HONEYMAN B.D. - Scavenging of thorium isotopes by colloids in the seawater of the Gulf of Mexico. Geochim. Cosmochim. Acta, $1992,56,3375-3388$.

[2] BYRNE R.H., KIM K. - Rare earth element scavenging in sea water. Geochim. Cosmochim. Acta, 1990, 54, 2645-2656.

[3] BRÜGMANN L., BERNARD P.C., VAN GRIEKEN R. - Geochemistry of suspended matter from the Baltic Sea. Mar Chem. 1992, 38, 303-323.

[4] BRUN-COTTAN J.C., AUGER R., LAMBERT C.E., CHESSELET R. - Sources and transport of suspended calcites in Pacific deep water. J. Mar. Res., 1991, 49, 543-564.

[5] DEGUELDRE C., LONGWORTH G., MOULIN V., VILKS P. - Grimsel colloid exercise. CEC Report EUR-12660 EN, 1990.

[6] DYMOND J. - Particles in the ocean. Oceanus, 1992, 60-67. 
[7] EREL Y., MORGAN J.J. - The effect of surface reactions on the relative abundances of trace metals in deep-ocean water. Geochim. Cosmochim. Acta, 1991, 55, 1807-1813.

[8] FARRINGTON J.W. - Mar. Chem, 1992, 39, 39-50.

[9] FISHER N.S., NOLAN C.V., FOWLER S.W. - Scavenging and retention of metals by zooplankton fecal pellets and marine snow. Deep-Sea Res., 1991, 38, 1261-1275.

[10] GUO L., COLEMAN C.H., SANTSCHI P.H. - The distribution of colloidal and dissolved organic carbon in the Gulf of Mexico. Mar. Chem, 1994, 45, 105-119.

[11] HONEYMAN B.D., BALIESTRIERI L.S., MURRAY J.W. - Oceanic trace metal scavenging : the importance of particle concentration. Deep-Sea Res., 1988, 35, 227-246.

[12] HONEYMAN B.D., SANTSCHI P.H. - The role of particles and colloids in the transport of radionuclides and trace metals in the oceans. In: Environmental particles (Buffe J., van Leeuwen H.P., Eds). Boca-Raton : Lewis Publishers, 1992, vol. 1, 379-423.

[13] JANNASCH H.W., HONEYMAN B.D., BALITRIERI L.S., MURRAY J.W. - Kinetics of trace element uptake by marine particles. Geochim. Cosmochim. Acta, 1988, 52, 567-577.

[14] JOHNSON B.D., KEPKAY P.E. - Colloid transport and bacterial utilisation of oceanic DOC. Deep-Sea Res., 1992, 39, 1855-869.

[15] KUMA K., SUZUKI Y., MATSUNAGA K. - Solubility and dissolution rate of colloidal $\gamma$ FeOOH in sea water. Water Res., 1993, 27, 651-657.

[16] LAVOIE D.M. - Computerized oceanic particle characterisation using heavy metal staining, SEM, EDXS and image analysis. Deep-Sea Res., 1992, 39, 1655-1668.

[17] LEPPARD G., BURNISON B., BUFFLE J. - Transmission electron microscopy of the natural organic matter. Anal. Chim. Acta, 1990, 232, 107-121.

[18] McCARTHY J.F., DEGUELDRE C. - Sampling and characterisation of colloids and particles in groundwater for studying their role in contaminant transport. In : Environmental particles (Buffle J, van Leuwen H.P., Eds). Roca Baton : Lewis Publishers, 1993, vol. 2, 247-315.

[19] MOORE R.M., HUNTER K.A. - Thorium adsorption in the ocean : reversibility and distribution amongst particle sizes. Geochim. Cosmochim. Acta, 1985, 49, 2253-2257.

[20] MOULIN V., OUZOUNIAN G. - Role of colloids and humic substances in the transport of radioelemnts through the geosphere. Appl. Geochem., 1992, 1, 179-186.

[21] NOMIZU T., MIZUIKE A. - Electron microscopy of submicron particles in natural waters : specimen preparation by centrifugation. Mikrochim. Acta, 1986, 1, 65-72.

[22] NIVEN S.E.H., MOORE R.M. - Thorium sorption in seawater suspensions of aluminum oxide particles. Geochim. Cosmochim. Acta, 1993, 57, 2169-2179.

[23] PERRET D., NEWMAN M.E., NEGRE J.C., CHEN Y., BUFFLE J. - Submicron particles in the Rhine river - I. Physico-chemical characterization. Water Res., 1994, 28, 91-106.

[24] PERRET D., LEPPARD G., MÜLlER M., BELZILE N., DE VITRE R., BUFFLE J. - Electron microscopy of aquatic colloids : non-perturbing preparation of specimens in the field. Wat. Res. 1991, 25, 1333-1343.

[25] SALBU B., BJORNSTAD H.E., SVAREN I., PROSSER S.L., BULMAN R.A., HARVEY B.R., LOVETT M.B. - Size distribution of radionuclides in nuclear fuel reprocessing liquids after mixing with seawater. Sci. Total Environ. 1993, 130/131, 51-63.

[26] SEMPERE R., CHARRIERE B., CAUWET G. - Importance of organic colloids in estuarine waters and continental margin. Ann. Inst. Oceanogr., Paris, 1993, 69, 167-171.

[27] SHOLKOVITZ E.R., LANDING W.M., LEWIS B.L. - Ocean particle chemistry : the fractionation of rare earth elements between suspended particles and seawater. Geochim. Cosmochim. Acta, 1994, 58, 1567-1579.

[28] WALSH I.D. - Large aggregation flux and fate at the seafloor: diagenesis during the rebound process. In: Deep-sea food chains and the global carbon cycle (Rowe G.T., Pariente V., Eds). Dordrecht : Kluwer, 1992, 365-373.

[29] WALSH I.D., GARDNER W.D. - A comparison of aggregate profiles with sediment trap fluxes. Deep-Sea Res., 1992, 39, 1817-1834. 
[30] WEI C.L., MURRAY J.W. - Temporal variations of ${ }^{234} \mathrm{Th}$ activity in the water column of Dabob bay : particle scavenging. Limnol. Oceanogr., 1992, 37 (2), 296-314.

[31] WELLS M.L., GOLDBERG E.D. - Occurrence of small colloids in sea water. Nature, 1991, $353,342-344$.

[32] WELlS M.L., GOLDBERG E.D. - Marine submicron particles. Mar. Chem., 1992, 40, 5-18.

[33] WELLS M.L., GOLDBERG E.D. - Colloid aggregation in seawater. Mar. Chem., 1993, 41, 353-358.

[34] WITHFIELD M., TURNER D.R. - The role of particles in regulating the composition of seawater. In: Aquatic surface chemistry - chemical processes at the particle-water interface (Stumm W., Ed). London : Wiley, 1987, 457-493. 